\title{
The Critical Role of Hospitalists for Successful Hospital-SNF Integration Hospitalists and Hospital/SNF Integration
}

\author{
John McHugh, PhD, MBA*; Patrick Shay, PhD²; Bradley Flansbaum, DO, MPH ${ }^{3}$
}

\begin{abstract}
${ }^{1}$ Department of Health Policy and Management, Mailman School of Public Health, Columbia University, New York, New York; ${ }^{2}$ Department of Health Care Administration, Trinity University, San Antonio, Texas; ${ }^{3}$ Department of Population Health, Geisinger Medical Institute, Danville, Pennsylvania.
\end{abstract}

n 2015, the Centers for Medicare and Medicaid Services (CMS) tied $42 \%$ of Medicare payments to a value-based model of care. ${ }^{1}$ Many of these models are designed to expand the scope of hospitals' accountability to include care provided to patients postdischarge (eg, readmission penalties, bundled payments, accountable care organizations). With such a significant change in organizational incentives, one would expect to see activity as it relates to hospital-skilled nursing facility (SNF) integration, potentially including shared risk among providers. ${ }^{2,3}$

Hospitals can choose from several different strategies when contemplating SNF integration, such as vertical integration with SNFs, which would involve acquiring and owning SNFs. However, despite the high level of incentive alignment and financial integration achieved through SNF acquisition, this strategy has not been widely adopted. Perhaps this is because hospitals can often attain a shorter length of stay and lower readmission rates without taking on the additional risk of owning a facility, except under particular market conditions. ${ }^{4}$ Hospitals can alternatively pursue virtual integration by developing preferred provider networks through contractual relationships or other formal processes, attempting to direct patients to SNF providers that have met predefined criteria, as described by Conway and colleagues in this issue of the Journal of Hospital Medicine ${ }^{\circledR}$. While hospitals have adopted this form of integration more widely than vertical integration, only those with additional financial motivations, such as those employing bundled payments, engaged in accountable care organizations (ACOs) or forward-thinking organizations preparing for looming global models of payment, have implemented such action. Finally, hospitals can focus on relational coordination through informal person-to-person communication and transition management. Given the high number of patients discharged to SNFs, the strategies above are not mutually exclusive, and enhanced relational coordination is most likely going to occur regardless of the type of - and perhaps even without-organizational-level integration.

For those hospitals choosing not to pursue integration

*Corresponding Author: John McHugh, PhD, MBA; E-mail: jpm2192@cumc. columbia.edu; Telephone: 212-304-5208.

Received: December 14, 2018; Revised: December 26, 2018;

Accepted: December 31, 2018

๑ 2019 Society of Hospital Medicine DOI 10.12788/jhm.3161 with SNFs, there are several reasons to maintain the status quo. First, hospitals have different interpretations of provider choice ("beneficiary freedom to choose"), whereby many do not believe they can provide information to patients outside of facility names and addresses. As such, they will refrain from developing a SNF network due to their interpretation of hazy federal rules. ${ }^{6}$ Second, it is possible that the incremental benefit of establishing a network is viewed by many hospitals as not worth the cost, measured by the time and effort required and the potential risk of not adhering to choice requirements. This could be especially true for hospitals without additional financial motivations, such as participation in an ACO or bundled payment program.

As the landscape continues to evolve, more successful systems will embrace a more concordant partnership with local and regional SNF providers, and several market factors will support the trend. First, the Medicare Payment Advisory Commission (MedPAC) is discussing the idea of choice in the context of postacute discharge, potentially leading to hospitals relaxing their strict interpretations of choice and the level of information provided to patients. ${ }^{7}$ Second, the evidence supports better patient outcomes when hospitals develop SNF networks. ${ }^{8,9}$ Finally, continued penetration of value-based payment models combined with CMS decisions regarding choice will continue to provide the additional motivation hospitals may need to change the cost-benefit calculation in favor of developing a network.

\section{IMPLICATIONS FOR HOSPITALISTS}

Traditionally, primary care physicians followed their patients through the acute- and postacute care continuum, but a variety of changes led to the growth of hospital medicine as fewer primary care physicians saw patients in the hospital. ${ }^{10,11}$ This shift has challenged efforts to ensure continuity of care across settings, especially since most hospitalists have ceded control of postdischarge placement to case managers and therapists. Further, there has been little incentive to connect hospitalists to any other component or provider along the range of care, and compensation models rarely, if at all, consider any accountability for patient outcomes outside the hospital. Several factors can change this reality for hospitalists.

First, as more providers adopt team-based care approaches and as alternative payment models expand the scope of accountability, hospitalists will become an even more central component of the risk evaluation process for hospitalized pa- 
tients as it relates to their discharge profile. This could mean that hospitalists are more involved in the postdischarge follow-up of patients sent home, to make sure patients adhere to discharge instructions. Alternatively, hospitalists may need to increase the level of physician-to-physician communication with SNF medical directors for patients discharged to SNF. This, in turn, could result in an increasing number of hospitalist groups recruiting SNFists to join their group or potentially assigning existing hospitalists or physician assistants to round on patients in the SNF. The 2018 Society of Hospital Medicine report showed an increase in activity among hospital medicine groups performing services in postacute-care facilities outside the hospital from $13 \%$ in 2016 to $25 \%$ in $2018 .{ }^{12}$ Similarly, a 2017 study in JAMA Internal Medicine reported a $48.2 \%$ increase in the number of physicians classified as SNFists from 2007 to $2014 . .^{13}$

Second, hospitalists will be more involved in the discharge planning process through internal interdisciplinary team communications. Whereas case managers and therapists owned the discharge planning process historically, new teams will include hospitalists, case managers, physical therapists, and pharmacists. System leaders will task them with identifying the appropriate discharge destination (eg, SNF, home health), finalizing the medication reconciliation, scheduling follow-up appointments, and completing a warm handoff.

Finally, as the field matures and hospitalists learn more about postacute-care connections, they will continue to be held more accountable for patient outcomes postdischarge. Many hospitalists have already connected to community providers through checklists and use evidence-based discharge programs like ProjectRed or Project BOOST.14,15 Organizations will need a similar strategy for SNFs, developing process measures, with the input of hospitalists, around those noteworthy areas that hospitalists can control. This will require greater alignment among constituents around overall organizational goals and, more importantly, entail the hospitalist to be attuned to overall patient goals beyond the care provided in the hospital setting.

As payment and care models continue to evolve, the status quo cannot be sustained. We anticipate that hospitalists will become more integrated into the patient discharge process, especially as it relates to discharge to SNFs before patients reconnect to their community physicians. Hospital systems will accelerate integration through the development of preferred SNF networks, and hospitalists stand to play a critical role in the success of these arrangements by enriching the benefits they create through these outward relationships.

For organizations engaged in embedded networks, they can realize gains via incentive alignment, trust, information transfer, mutual support, and coordination through virtual integration, without requiring vertical ownership.,16 Thus, the opportunity exists for hospitalists to be critical drivers of network success, serving as intermediaries from which information, collaboration, and shared problem-solving flow between hospitals, SNFs, patients, and the entire care team. Opportunities to rebuild our system are long past; however, like all changing sectors in healthcare, the disaggregate acute and postacute settings must move in lockstep. Hospitals and postacute care facilities must find ways to alter their thinking to eradicate the obstructive and injurious invisible wall.

Disclosures: The authors have nothing to disclose.

\section{References}

1. Catalyst for Payment Reform. CPR Scorecard on Medicare Payment Reform 2015.

2. Mick S, Shay P. Accountable care organizations and transaction cost economics. Med Care Res Rev. 2016;73(6):649-659. doi: 10.1177/1077558716640411.

3. Shay P, Mick S. Post-acute care and vertical integration after the Patient Protection and Affordable Care Act. J Healthc Manag. 2013;58(1):15-27.

4. McHugh J, Zinn J, Shield R, et al. Strategy and risk-sharing in hospital-postacute care integration. Health Care Manage Rev. 2018. doi: 10.1097/ HMR.0000000000000204.

5. Conway S, Parekh A, Hughes A, et al. Post-acute care transitions: developing a skilled nursing facility collaborative within an academic health system. J Hosp Med. 2019;14(3):174-177. doi: 10.12788/jhm.3117.

6. Tyler D, Gadbois E, McHugh J, Shield R, Winblad U, Mor V. Patients are not given quality-of-care data about skilled nursing facilities when discharged from hospitals. Health Aff. 2017;36(8):1385-1391. doi: 10.1377/ hlthaff.2017.0155.

7. Medicare Payment Advisory Commission. Encouraging Medicare Beneficiaries to Use Higher Quality Post-Acute Care Providers. Washington, DC: MedPAC; 2018

8. McHugh J, Foster A, Mor V, et al. Reducing hospital readmissions through preferred networks of skilled nursing facilities. Health Aff. 2017;36(9):15911598. doi: 10.1377/hlthaff.2017.0211.

9. Rahman M, Foster A, Grabowski D, Zinn J, Mor V. Effect of hospital-SNF referral linkages on rehospitalization. Health Serv Res. 2013;48(6):1898-1919. doi: 10.1111/1475-6773.12112.

10. Wachter R, Goldman L. Zero to 50,000 - The 20th anniversary of the hospitalist. N Engl J Med. 2016;375(11):1009-1011. doi: 10.1056/NEJMp1607958.

11. Kripalani S, Jackson A, Schnipper J, Coleman E. Promoting effective transitions of care at hopsital discharge: A review of key issues for hospitalists. $J$ Hosp Med. 2007;2(5):314-323. doi: 10.1002/jhm.228.

12. Society of Hospital Medicine. 2018 State of Hospital Medicine Report. Philadelphia: Society of Hospital Medicine; 2018. 2018 SHM Report.

13. Teno J, Gozalo P, Trivedi A, Mitchell S, Bunker J, Mor V. Temporal trends in the numbers of skilled nursing facility specialists from 2007 through 2014. JAMA Intern Med. 2017;177(9):1376-1378. doi: 10.1001/jamainternmed.2017.2136.

14. Boston University Medical Center. Project RED Re-Engineered Discharge. Project RED. Available at: https://www.bu.edu/fammed/projectred/. Accessed Dec 9, 2018.

15. Hansen L, Greenwald J, Budnitz T, et al. Project BOOST: effectiveness of a multihospital effort to reduce rehospitalization. J Hosp Med. 2013;8:421-427. doi: 10.1002/jhm.2054

16. Uzzi B. The sources and consequences of embeddedness for the economic performance of organizations: the network effect. Am Sociol Rev. 1996:674698. doi: 10.2307/2096399 